\title{
Differences in Inflammation, Apoptosis and Tissue Hypo- perfusion Indicators Caused by Using Two Different Gauges in Cecal Ligation and Puncture-induced Rat Models of Sepsis
}

Tina Didari ( $\nabla$ didari.tina@gmail.com )

Tehran University of Medical Sciences https://orcid.org/0000-0002-6116-8451

Maryam Baeeri

Tehran University of Medical Sciences

Mahban Rahimifard

Tehran University of Medical Sciences

Shilan Mozaffari

Tehran University of Medical Sciences

Hamed Haghi-Aminjan

Ardebil University of Medical Sciences

Mojtaba Mojtahedzadeh

Tehran University of Medical Sciences

Atabak Najafi

Tehran University of Medical Sciences

Research

Keywords: Sepsis, Cecal Ligation and Puncture (CLP), Inflammation, Oxidative Stress, Needle Gauge, Rat

Posted Date: August 12th, 2020

DOl: https://doi.org/10.21203/rs.3.rs-54719/v1

License: (c) (i) This work is licensed under a Creative Commons Attribution 4.0 International License. Read Full License 


\section{Abstract}

Background: Rodent Cecal Ligation and Puncture (CLP) models, which mimic sepsis in humans. Gauges needle size during CLP related to cytokine storm, inflammation and organ failure. This study focus on precise and low cost various biochemical markers to trace sepsis severity in cardiopulmonary system and blood after CLP with gauge needle size -18 (G-18) and -21 (G-21) in rats.

Results: For the purpose of this study, 18 male Wistar rats were assigned into three groups: CLP procedure with G-18 needle, CLP procedure with G-21 and sham. Following the procedure, oxidative stress markers, blood markers, apoptosis indicators, gene expression of autophagy and cellular hypoxia and histopathological assessments were carried out in the cardiopulmonary tissue and blood 24 hours after CLP. All measured factors were increased in both G18 and G-21 gauge groups in comparison with the sham. Moreover, 24 hours after CLP, inflammatory markers, blood profile, lactate level, pro-inflammatory cytokines, caspases, gene expression of cellular homeostasis and autophagy in samples were more pronouncedly increased in the G-18 group in comparison with the G-21.

Conclusions: This experiment provides a significant association of inflammation and cytokine storm with anti-oxidant property, apoptotic condition and gene expression of tissue and blood infection related to severity organ damage were increased with G-18 compare to G-21. Our study has identified cost-effective indicators to evaluate organ failure during sepsis. This should be considered by all investigators using G-18 in comparison with G-21 to induce severe sepsis.

\section{Introduction}

Sepsis is a common cause of inpatient mortality in both genders and all age groups, particularly in the intensive care units (ICU) of hospitals. Annually, more than 19 million people are affected by sepsis (with a mortality rate as high as $18 \%$ ) worldwide, and approximately $50 \%$ ICU services. $(1,2)$ This deadly condition ensues an infection-caused inflammatory process in the body. In sepsis, the cause of death is generally multi-organ dysfunction, and in about $50 \%$ of the affected individuals, the cardiovascular system is inflicted. (3) Despite the recent decrease in its mortality rate though advancements in antibiotics and intensive care equipment $(1,2)$, the incidence of sepsis is increasing worldwide, possibly due to ageing populations and the increased incidence of co-morbid conditions in advanced ages. (4)

Sepsis-induced shock is associated with multi-organ failure with a mortality rate of $40 \%$, and the most frequently affected organs are the cardiovascular and respiratory systems. (3) Although the precise mechanisms of sepsisinduced cardiomyopathy are not yet fully elucidated, it suggested that severe inflammation, metabolism insufficiencies, and defective beta-adrenergic response may be involved in this process.(4) Moreover, during sepsis, mainly due to hyperlactatemia, both systolic and diastolic cardiac impairment occur with the consequence of mitochondrial damage in the cardiac tissue (5). Pulmonary complications of sepsis develop as a result of the cumulative effect of circulating cellular elements, soluble inflammatory mediators, and cytokines on the pulmonary tissues. (6) The main underlying mechanism of lung injury during sepsis is demonstrated to be acute alveolar infiltration and changes in respiration ratio (7).

Considering its high fatality rate, sepsis leaves little opportunity for clinical research in the field. Therefore, CLP procedure which is considered as the gold standard model of human sepsis provides an invaluable opportunity for investigation into different aspects of sepsis. In this procedure in the animal model of the disease, an induced results in the secretion of intestinal poly-microbial flora into the abdomen, a contamination followed by vascular dissemination of the micro-organisms. This leads to systemic presentations very similar to those of sepsis in humans. (8) 
CLP-induced damages (and sepsis in humans) stimulate the immune system, activate oxidative stress pathways, increase caspase levels (due to apoptosis), and lead to the expression of autophagy and hypoxia-related genes (9). Reactive oxygen species (ROS) are major signaling molecules involved in the oxidative stress response. In sepsis, the excessive accumulation of ROS impairs cellular homeostasis, and this leads to oxidative stress and mitochondrial dysfunction. This oxidative stress process also promotes autophagy, a defensive cytoprotective process for recycling waste organelles and other intracellular material following cellular damages.(10) Consequently, indicators of oxidative stress and autophagy processes have been widely used to investigate the intensity of sepsis induced the CLP procedure.(11) Similarly, measurement of the enzyme AMP-activated protein kinase is extensively employed for assessment of the severity of sepsis (12).

It is demonstrated that the size of the needle used in the CLP procedure can be influential in different septic outcomes such as mortality, cytokines' concentrations, apoptosis, lactic acidosis, and autophagy. (13)

It is demonstrated that the size of the needle used in the CLP procedure can be influential in different septic outcomes such as mortality, cytokines concentrations, apoptosis, lactic acidosis, and autophagy. Previous studies showed that G-18 and G-21 induced sepsis more efficiently compared to other gauge groups $(6,11,12)$.

There have been little experiments and limited set of factors affecting multi-organ dysfunction syndrome (MODS), with G-18 and G-21. New inexpensive and simple indicators have required to modify needle size related to MODS during CLP. In this study, we aimed to find and compare precise, inexpensive and new biomarkers in heart, lung and blood profile after CLP induced sepsis with G-18 and G-21.

In this study, on the presumption that these processes may underlie differences in organ dysfunction and death, we aimed to measure and compare inflammatory markers and expression of hypoxia and autophagy-related genes in cardiac and pulmonary tissues of CLP models of sepsis induced by two different gauges (18 and 21).

\section{Materials And Methods}

\section{Animals}

Adult male Wistar rats (3-4) months old (220-310 g) were procured from the Animal Breeding House of Faculty of Pharmacy of TUMS. Animals were kept according to the animal standard care facility under controlled temperature (23 $\pm 10 \mathrm{C}), 12 \mathrm{~h}$ light/dark cycle, $55 \pm 10 \%$ humidity and ad libitum feed. The protocol of the study was approved by the institute ethical committee under code number IR.TUMS.VCR.REC.1396.2341 and Reporting of in Vivo Experiments (ARRIVE) Guideline.

\section{Reagents}

For High mobility group box 1 (HMGB1) and TNF-a, ELISA kits were procured from ZellBio GmbH (Ulm, Germany) and Diaclone (France), respectively. Lactate isolation kit we used was produced from ZellBio GmbH (Ulm, Germany). RNase solution, iScript cDNA synthesis kit, and propidium iodide were manufactured by Sigma-Aldrich GmbH (Munich, Germany). Other chemicals not specifically mentioned were all purchased from Sigma-Aldrich GmbH (Munich, Germany).

\section{Animal groups}

Eighteen rats were randomly allocated into 3 groups consisting of:

Group $1(n=6)$; Sham group (which underwent exactly the same operation without the CLP procedure) 
Group 2 ( $n=6)$; CLP group with G-18 (which underwent CLP procedure and two punctured with gauge 18)

Group $3(n=6)$; CLP group with G-21 (which underwent CLP procedure and two punctured with gauge 21)

\section{The procedure}

Adult male Wistar rats first underwent CLP as described in details elsewhere (14). Rats were anesthetized with an intraperitoneal dose of ketamine $(80 \mathrm{mg} / \mathrm{kg})$ and xylazine $(10 \mathrm{mg} / \mathrm{kg})$. Then, a 3-cm midline laparotomy was performed in order to expose the cecum and adjoining intestine. The cecum was ligated with a 3.0 silk suture at its base right below the ileocecal valve, and was perforated two times using 18-or 21-gauge according to the group animals were assigned to. Dimensions of the needles are as follows: Gauge $18=1.270 \mathrm{~mm}$, Gauge $21=0.8192 \mathrm{~mm}$. Next, the cecum was replaced in the peritoneal cavity and the incision was closed using 4.0 silk sutures. Following the operation, animals were hydrated though intravenous administration of an isotonic saline solution ( $50 \mathrm{~mL} / \mathrm{kg}$ s.c.), returned to a cage in a period of 24 hours (15). CLP procedure was assessed with Murine Sepsis Score (MSS) by an experienced technician (16).

\section{Sample preparation}

24 hours following the procedure, the animals were anesthetized with overdoses of ketamine and xylazine (80-100 $\mathrm{mg} / \mathrm{kg}$ and $5-10 \mathrm{mg} / \mathrm{kg} \mathrm{IP}$ ), and were sacrificed. Blood specimens were then collected from the hearts of the rats and subsequently divided into serum separator and ethylenediaminetetraacetic acid (EDTA) tubes for analysis of related blood markers. The tissues were harvested and washed with saline then put into two parts for further histopathological and biochemical assessments. Biochemical samples were stored as frozen samples at $-80{ }^{\circ} \mathrm{C}$ refrigerator, and pathological specimens were deposited and fixed in $10 \mathrm{~mL}$ of $10 \%$ formalin.

\section{Determination of oxidative stress biomarkers}

\section{ROS assay}

Tissue samples were all homogenized and centrifuged accordingly. Subsequently, they were stored in $75 \mu \mathrm{L}$ extraction buffer, mixed with $80 \mu \mathrm{L}$ of assay buffer, and kept for $30 \mathrm{~min}$ at $37^{\circ} \mathrm{C}$. Production of ROS was absorbed by $2^{\prime}, 7^{\prime}$ dichlorofluorescein diacetate (DCF-DA) as fluorogenic reagent. Identification of the absorbance change of DCF-DA was identified by means of ELISA fluorometer (Biotec, Tecan U.S.) with maximum excitation (488 nm) and emission (529 $\mathrm{nm}$ ) spectra for an hour.

\section{LPO assay}

Malondialdehyde (MDA) is generally considered as the final by-product of lipid peroxidation (LPO) during generation process of ROS. In the current study, Thiobarbituric acid reactive substances (TBARS) assay were employed for detection of the complex of MDA and Thiobarbituric Acid (TBA). MDA concentration were reported at $532 \mathrm{~nm}$ with spectrophotometer. As described in details elsewhere, the amount of MDA formed by tissue samples was reported as $\mu \mathrm{M} / \mathrm{mg}$ protein (17).

\section{Total antioxidant power (TAP) assay}

FRAP test is widely used for measurement of antioxidant potential of samples. Accordingly, for deoxidizing the Fe3+ TPTZ (2, 4, 6-tris-(2-pyridyl)-s-triazin) to Fe2+ , $1 \mathrm{~mL}$ of Tris-EDTA buffer was added to $50 \mu \mathrm{L}$ of samples, and, consequently, the absorbance was measured at $593 \mathrm{~nm}$ (18). 
Assay of total thiol molecules (TTM)

Total thiol molecules is defined as antioxidant content of samples (19). For thiol assessment, interaction of TTM with Ellman's reagent was estimated in maximum peak at $412 \mathrm{~nm}(20)$.

\section{Myeloperoxidase (MPO) activity}

The homogenization of tissue samples were carried out in $50 \mathrm{mM}$ potassium buffer $(\mathrm{pH} 6.0)$ containing $0.5 \%$ hexadecyltrimethylammonium bromide (HTAB). Subsequently, the samples were centrifuged $\left(30000 \mathrm{~g}, 15 \mathrm{~min}, 4^{\circ} \mathrm{C}\right)$ and $100 \mu \mathrm{l}$ of the supernatant was mixed with phosphate buffer $(\mathrm{pH} 6,50 \mathrm{mM})$ containing $0.167 \mathrm{mg} / \mathrm{ml}$ of odianisidine dihydrochloride and $0.0005 \% \mathrm{H}_{2} \mathrm{O}_{2}$. Change of absorbance was recorded through spectrophotometric analysis at $460 \mathrm{~nm}$ five minutes later. The expression of MPO activity was defined as the change of enzyme absorbance during conversion of $1 \mu \mathrm{mol}$ of $\mathrm{H}_{2} \mathrm{O}_{2}$ to $\mathrm{H}_{2} \mathrm{O} / 1 \mathrm{~min}$ at $37^{\circ} \mathrm{C}$, as described in details elsewhere (21). Unit per mg of tissue protein was considered as the index of MPO activity.

\section{Caspase -3 and -9 activation in the cardiac and lung tissues}

Colorimetric assays were utilized for measurement of the caspases-3 and -9 through specific identification of particular amino acid sequences. Briefly, the application of chromophore r-nitroaniline (qNA) produces a yellow color which can be detected by spectrophotometry at $405 \mathrm{~nm}$. For this purpose, tissue samples were degraded by lyses buffer and incubated for $10 \mathrm{~min}$ on ice. Moreover, the caspase buffer which consisted of $100 \mathrm{mM}$ of caspase-3 and -9 specific substrate along with total cell lysates were incubated at $37^{\circ} \mathrm{C}$ for $4 \mathrm{~h}$. The standard absorbance of caspase-3 and -9 was detected at $405 \mathrm{~nm}(22)$.

\section{Lactate levels in tissue and serum samples}

\section{Tissue samples}

According to the Manufacture's protocol lactate assay was performed for analysis of all samples. In short, tissue samples $(100 \mathrm{mg}$ ) were homogenized in $8 \%$ perchloric acid and lactate level evaluated with using a standard curve.

\section{Serum samples}

After allowing the serum separator tube to clot for 10 minutes at $37^{0} \mathrm{c}$, samples centrifuged (at $3000 \mathrm{~g}$ for 20 minutes), then supernatants were collected. The lactate levels of serum samples were determined using a standard curve and reported as $\mathrm{mmol} / \mathrm{L}$ of serum protein.

\section{Assessment of pro-inflammatory cytokines}

\section{Measurement of TNF-a levels}

The quantity of TNF-a in test samples was examined by a rat-specific TNF-a ELISA kit (Diaclone, France). According to instruction of the kit, test samples and standards were added to the wells containing antibodies. After washing, biotinylated anti-rat TNF-a antibody and HRP conjugated streptavidin were added to each well, samples were rewashed, and TMB and stop solutions were mixed to the wells respectively. The conversion of blue to yellow color, which is demonstrated to be proportional to the TNF-a level, was estimated at $450 \mathrm{~nm}$.

\section{Assessment of HMGB1 levels}


The quantity of HMGB1 in test samples was examined by means of a rat-specific HMGB1 ELISA kit. Test and standards samples were added to the wells containing immobilized antibodies. Following washing, biotinylated antirat HMGB1 antibody and HRP conjugated streptavidin were added to the wells. Then samples were rewashed and chromogen and stop solutions were added to the wells. A color change (from blue to yellow) in proportion with the HMGB1 levels was assessed at $450 \mathrm{~nm}$.

\section{Gene expression evaluation}

For Quantitative Real-time PCR (qRT-PCR), $1 \mu \mathrm{g}$ of total RNA was reverse transcribed to cDNA using a Primescript RT reagent kit (TAKARA, Japan). qRT-PCR was performed using the Step one plus ABI system (Applied Biosystems). qRTPCR was carried out under the following conditions: $30 \mathrm{~s}$ at $95^{\circ} \mathrm{C}$ for 1 cycle, 40 cycles of $95^{\circ} \mathrm{C}$ for $5 \mathrm{~s}, 60{ }^{\circ} \mathrm{C}$ for $34 \mathrm{~s}$ and $72{ }^{\circ} \mathrm{C}$ for $45 \mathrm{~s}$. One cycle of $30 \mathrm{~s} 72^{\circ} \mathrm{C}$ was done finally to allow final extension (23). The sequences of primers used for qRT-PCR are presented below;

For Glyceraldehyde-3-phosphate dehydrogenase (gene symbol: GAPDH)

sense primer (5'- AGTCTACTGGCGTCTTCACC -3')

antisense primer (5'- CCACGATGCCAAAGTTGTCA -3')

For AMP-activated catalytic subunit alpha 1 (AMPK) (gene symbol: Prkaa1)

sense primer (5'- CCGTCTTATAGTTCAACCAT-3')

antisense primer (5'- TTCGTTCATTATTCTCCTGTT-3')

For Microtubule-associated protein 1 light chain 3 beta (LC3Ilb) (gene symbol: Map1lc3b)

sense primer (5'- CAGTGATTATAGAGCGATACA-3')

antisense primer (5'- GCCTTCTAATTATCTTGATGAG -3')

\section{Blood samples parameters}

\section{Whole blood assessment}

Peripheral blood samples were collected for complete blood count (CBC). The CBC blood samples were collected into EDTA tubes. Hematologic parameters were analyzed 30 mins following sample collection using a hematology analyzer (Sysmex). The counts for lymphocyte $\left(10^{3} / \mu \mathrm{L}\right)$ and platelets $\left(10^{3} / \mu \mathrm{L}\right)$ and mean platelet volume $(\mathrm{MPV})(\mathrm{fL})$ were recorded and platelet to lymphocyte ratio (PLR) were calculated, using the results.

\section{Blood glucose levels}

24 hours after the CLP procedure, blood samples were collected by skilled personnel using the routine technique of tailtip amputation. Then the ACCU-CHECK Compact Plus ${ }^{\circledR}$ (Roche Diagnostics, Japan) glucometer were employed for measurement of blood glucose levels in 5 seconds.

\section{Histopathological studies}

The animals were euthanized 24 hours after the procedure, and heart and lung tissues were isolated and fixed in the $10 \%$ neutral buffered formalin (NBF, pH 7.26) for 48 hours, and then were embedded in paraffin. The 5- $\mu \mathrm{m}$ thick 
sections were then made ready for staining with hematoxylin and eosin (H\&E). Then the histological slides were observed using light microscope (Olympus BX51, Japan). Any suspected changes such as acute or chronic inflammatory response, congestion, hemorrhage or hyperemia, necrosis was investigated in different samples.

\section{Statistical analysis}

The results were presented as the mean \pm standard error of the mean (SEM). One-way analysis of variance (ANOVA) and Tukey's multi-comparison tests were applied with the degree of significance set at $(P<0.05)$.

\section{Results}

\section{Oxidative stress and anti-oxidant parameters}

ROS levels were raised significantly in lung tissue in CLP-G18 and CLP-G21 compared to the sham group ( $P<0.01$ and $\mathrm{P}<0.05$, respectively). Moreover, ROS production in heart tissue was increased significantly in CLP-G18 compared to sham group $(P<0.001)$. Likewise, another important finding was that CLP-G21 significantly decreased the amount of ROS compared to CLP-G18 $(P<0.001)$.

A significant increase in the levels of MDA in the lung and heart tissues were observed in CLP-G18 in comparison the sham group $(P<0.01)$. Moreover, the LPO level was diminished in the CLP-G21 group compared to the CLP-G18 ( $<<0.01$ and $\mathrm{P}<0.05$, respectively) in two different tissues.

Tissue FRAP in lung samples was decreased significantly in CLP-G18 compared to sham group $(P<0.001)$, but significant reduction of FRAP was only observed in heart tissue with both gauges (CLP-G18 and CLP-G21) compared to sham group $(P<0.001$ and $P<0.01$, respectively). Likewise, CLP-G21 significantly enhanced FRAP Levels in lung and tissue compared to CLP-G18 group ( $p<0.05$ and $p<0.001$, respectively).

TTM level in lung tissue was significantly reduced in CLP-G18 and CLP-G21 groups compared to sham group ( $P<0.01$ and $\mathrm{P}<0.05$, respectively), TTM level in heart tissue was significantly declined in CLP-G18 and CLP-G21 compared to sham group $(P<0.001$ and $P<0.01$, respectively). The oxidative stress parameters are presented in Table 1.

Table 1. Effect of gauge 18 and gauge 21 on Cecal ligation and puncture (CLP) on biochemical markers in heart and lung tissues. 


\begin{tabular}{|c|c|c|c|c|c|c|}
\hline & \multicolumn{3}{|l|}{ Heart tissue } & \multicolumn{3}{|l|}{ Lung tissue } \\
\hline & Sham & CLP-G18 & CLP-G21 & Sham & CLP-G18 & CLP-G21 \\
\hline $\begin{array}{l}\text { ROS(unit/mg } \\
\text { protein) }\end{array}$ & $0.66 \pm 0.05$ & $1.21 \pm 0.08^{\star \star \star}$ & $0.77 \pm 0.04^{\# \# \#}$ & $0.27 \pm 0.03$ & $0.98 \pm 0.16^{* \star}$ & $0.84 \pm 0.17^{\star}$ \\
\hline LPO $(\mu \mathrm{M})$ & $63.71 \pm 2.10$ & $96.51 \pm 5.95^{* \star}$ & $83.58 \pm 6.25^{*}$ & $76.51 \pm 6.86$ & $108.39 \pm 5.68^{* *}$ & $80.308 \pm 3.40^{\# \#}$ \\
\hline $\operatorname{FRAP}(\mu \mathrm{M})$ & $275.9 \pm 6.31$ & $213.57 \pm 4.28^{\star \star \star}$ & $\begin{array}{l}251.9 \pm 1.84^{* \star} \\
\# \#\end{array}$ & $284.23 \pm 6.16$ & $239.57 \pm 7.30^{\star \star \star}$ & $268.74 \pm 5.14^{\#}$ \\
\hline $\begin{array}{l}\text { TTM } \\
(\mu \mathrm{mol} / \mathrm{mg} \\
\text { protein })\end{array}$ & $0.96 \pm 0.11$ & $0.35 \pm 0.08^{\star \star \star}$ & $0.41 \pm 0.06^{*}$ & $1.13 \pm 0.20$ & $0.42 \pm 0.10^{\star \star}$ & $0.61 \pm 0.06^{*}$ \\
\hline $\begin{array}{l}\text { MPO (unit/gr } \\
\text { tissue) }\end{array}$ & $77.83 \pm 3.64$ & $97.64 \pm 6.07^{*}$ & $89.41 \pm 4.96$ & $75.97 \pm 7.50$ & $111.7 \pm 7.66^{* *}$ & $103.16 \pm 3.78^{\star}$ \\
\hline $\begin{array}{l}\text { Caspase-3 } \\
\text { activity(\% of } \\
\text { content) }\end{array}$ & $\begin{array}{l}99.85 \pm \\
4.26\end{array}$ & $99.85 \pm 4.26$ & $80.07 \pm 6.59^{\#}$ & $\begin{array}{l}100.12 \pm \\
2.52\end{array}$ & $138.29 \pm 8.97^{\star \star}$ & $\begin{array}{l}107.31 \pm \\
5.96^{\# \#}\end{array}$ \\
\hline $\begin{array}{l}\text { Caspase-9 } \\
\text { activity(\% of } \\
\text { content) }\end{array}$ & $\begin{array}{l}99.82 \pm \\
7.02\end{array}$ & $124.88 \pm 3.16^{*}$ & $\begin{array}{l}103.77 \pm \\
5.79^{\#}\end{array}$ & $99.85 \pm 4.62$ & $\begin{array}{l}131.44 \pm \\
11.10^{*}\end{array}$ & $139.45 \pm 7.82^{* *}$ \\
\hline
\end{tabular}

ROS (reactive oxygen species), LPO (lipid peroxidation), FRAP (ferric reducing antioxidant power), TTM (total thiol molecules), MPO (myeloperoxidase), Caspase- 3 and -9 assay. Results are expressed as mean \pm SEM for six animals in each group.

***: significant difference from sham group at $\mathrm{P}<0.001$,

**: significant difference from sham group at $\mathrm{P}<0.01$,

*: significant difference from sham group at $\mathrm{P}<0.05$,

\#\#\#: significant difference from CLP-gauge18 group at $\mathrm{P}<0.001$,

\#\#: significant difference from CLP-gauge18 group at $\mathrm{P}<0.01$,

\#: significant difference from CLP-gauge18 group at $\mathrm{P}<0.05$.

\section{Myeloperoxidase activity}

The result of MPO assay showed that a significant elevation was increased in lung tissue of CLP-G18 and CLP-G21 compared to sham group ( $P<0.01$ and $P<0.05$, respectively). However, the MPO level of CLP-G18 group in heart tissue was elevated significantly $(P<0.05)$ compared to sham and CLP-G21 groups (Table 1$)$.

\section{Caspase 3 and Caspase 9}

The result of enzyme activities of caspase-3 and caspase- 9 in lung tissue indicate that there was a significant difference between CLP-G18 and sham group ( $P<0.01$ and $p<0.05$, respectively). This figure shows a decrease of caspase 3 activity in CLP-G21 compared to CLP-G18 $(P<0.01)$. The results obtained from the caspase activity in heart tissue had no effect on the level of caspase-3 in CLP-G18 and CLP-G21 in comparison with the sham group, but the 
activity of caspase-9 was increased in CLP-G18 compared to sham group $(\mathrm{P}<0.05)$. A significant reduction in the level of caspase-9 activity was observed in CLP-G21 compared to CLP-G18 group $(P<0.05)$ (Table 1$)$.

\section{Lactate levels}

Lactate production, a reliable marker of tissue hypoperfusion was increased in CLP-G18 and CLP-G21 groups in lung $(P<0.001$ and $P<0.01)$ and heart tissues $(P<0.001)$ compared to the sham group. The blood lactate level raised in CLPG18 compared to sham groups $(P<0.001)$. Moreover, no significant differences were found between CLP-G21 and sham group (Fig. 1).

\section{Proinflammatory cytokines}

TNF-a which is an early modulator of inflammation during sepsis was found to be significantly increased in CLP-G18 and CLP-G21 $(P<0.001)$ in comparison with the sham group in lung samples. The levels of TNF-a in heart tissue was increased in CLP-G18 and CLP-G21groups compared to the sham group $(P<0.001$ and $P<0.05$, respectively) (Fig. 2A).

The amount of HMGB1, a late phase inflammatory biomarker, was significantly elevated in CLP-G18 and CLP-G21 groups in comparison with the sham group $(\mathrm{P}<0.001)$ in both lung and heart samples (Fig. $2 \mathrm{~B})$.

\section{Quantitative Real-time PCR}

The expression of AMPK as the stress protein in lung tissue was significantly increased in CLP-G18 and CLP-G21 groups compared to the sham group (1.77-fold and 2.23-fold, respectively). In addition, the mRNA expression level of AMPK in CLP-G18 and CLP-G21 groups of heart tissue increased significantly compared to the sham group (3.66-fold and 1.79-fold, respectively). The level of AMPK expression has been downregulated in CLP-G21 compared to CLP-G18 in lung and heart samples ( $P<0.01$ and $P<0.001$, respectively) (Fig. 3A).

The level of LC3Ilb gene expression as autophagy gene in lung tissue raised in CLP-G18 and CLP-G21 groups compared to the control group (4.38-fold and 2.36-folds, respectively). The analysis of lung tissue data showed that the expression level of LC3Ilb in the CLP-G21 group has been significantly reduced compared to the CLP-G18 group $(P<0.001)$. Likewise, the mRNA expression level of LC3Ilb in CLP-G18 and CLP-G21 groups significantly elevated compared to the sham group (5.86-fold and 5.3-fold, respectively) in heart tissue sample (Fig. 3B).

\section{Mean platelet volume (MPV) and platelet to lymphocyte ratio (PLR)}

Overall platelet function and inflammation in blood were measured in MPV and PLR, respectively. The levels of MPV and PLR were raised significantly in CLP-G18 compared to the sham group $(P<0.01)$ (Fig.4A, 4B). Also, there was a significant reduction in MPV and PLR in CLP-G21 compared to CLP-G18 group ( $P<0.05$ and $P<0.01$, respectively).

\section{Blood glucose levels}

Blood glucose analysis revealed a significant reduction in CLP-G18 and CLP-G21 compared to the sham group $(\mathrm{P}<0.001$ and $\mathrm{P}<0.05$, respectively) (Fig. $4 \mathrm{C})$.

\section{Histopathological analysis}

All the samples were visualized by an independent reviewer. The results of each sample have reported as below:

Micrographs of lung and heart in sham group showed normal structure without any histopathological findings. Histopathological evaluation of lung and heart tissues in CLP-G21 group showed a close similarity to sham group with 
normal structure and organization of lung and heart tissue. Micrographs of the lung in CLP-18 group showed the various degree of lung injury such as pneumocyte type II (hyperplasia), peribronchiolar inflammation and hyperemia. The thickness of alveolar septa significantly increased in comparison to normal area . However, lung inflammation was verified by the presence inflammatory cells (lymphocyte) and edema. The inflammatory response was confirmed by lymphocyte counts in five random high-magnification $(\times 1,000)$ fields per slide. $7.5 \pm 0.12$ lymphocyte/field was present in CLP samples. Moreover, the histopathological evaluation of the heart sample in CLP-G18 group showed myocardial cell necrosis (Fig. 5).

\section{Discussion}

We designed and carried out the current study to illuminate differences in cardiac and pulmonary tissues damages following CLP procedure in rats. To our best knowledge, this is the first study investigating the differences two needle sizes, namely 18 and 21, cause in cellular and molecular sepsis mechanisms such as oxidative stress, expression of homeostatic-autophagic related genes, proinflammatory cytokines, apoptosis, tissue perfusion and hematologic parameters in male Wistar rats. Findings of our study demonstrated that 24 hours after the CLP procedure, we observed that inflammatory markers, blood markers, blood and tissue lactate levels, pro-inflammatory cytokines, caspases, and gene expression of cellular homeostasis and autophagy in samples were more pronouncedly increased in the G-18 group in comparison with the G-21. This should be taken into consideration for development and execution of related protocols to increase the accuracy of the results and avoid waste of resources.

Oxidative stress is a major indicator of sepsis induced by CLP procedure. Oxidative stress pathways and ROS generation are consisted of several components such as: increased LPO levels, alternated metabolic gene expression, and increased MPO levels during the procedure $(24,25)$. In this regard, many consider MPO as a major indicator of the neutrophil infiltration process. (25-30) It is demonstrated that sepsis induced by both gauge 18 and gauge 21 can elevate ROS levels in the liver, colon and kidney 16 to 24 hours following the procedure (31-34). In addition, 6 to 48 hours after the procedure, LPO level, increases in the lung, ileum, diaphragm, heart, brain and kidney (regardless of the gauges used) (26-30, 34-41). Congruently, the findings of our study demonstrated that 24 hours after the CLP procedure, in both cardiac and pulmonary tissues, oxidative stress markers increased, whereas the levels of antioxidant indicators reduced in comparison with the sham group. Interestingly, we observed that this effect was more accentuated when gauge 18 needles were used in comparison with gage 21 . Similarly,

As regards blood markers (due to our limited resources), we applied low cost, quick and available hematologic tests to assess MPV, PLR and glucose level of blood samples to determine sepsis. MPV is an indicator of platelet function and endothelial disruption which is increased in the early and late phase of sepsis (42-45). Canine model of sepsis have demonstrated higher levels of MPV in the septic group in comparison with the control animals (46). In line with such observations, our findings demonstrated that MPV levels were elevated in the late phases of the procedure with the G18 needles. It is widely reported that blood glucose levels are elevated in early phase of CLP, whereas they decrease in the in the late phases (47-50). In this regard, we noticed that blood glucose levels were more pronouncedly reduced in the CLP-G18. This observation is in line with those reporting that PLR is elevated in hospitalized patients with end stage renal disease and nosocomial infection $(51,52)$. Thus, it can be claimed that current study was the first to report that the raise on PLR levels is more prominent in the CLP-G18 murine models. This should be seriously considered in design and development of related protocols in this field.

Lactate level is generally considered as a reliable indicator of tissue hypo-perfusion, hypoxia and altered microcirculation in CLP models of sepsis. Previous studies have separately reported raised lactate levels following CLP-G18 in blood, ileum and liver, and noted the elevation of the lactate levels in lung and colon following CLP

Page $10 / 20$ 
procedure with $\mathrm{G} 22$ needles $(25,27,49,53-55)$. In this regard, we observed that the blood and tissue lactate levels increased more intensely following G18 CLP procedure in comparison with CLP-G21.

Regarding proinflammatory cytokines, it is demonstrated that the blood levels of these indicators of inflammation, particularly TNF- $a$ and HMGB1, is altered during CLP procedure. (56-59) Previous reports have demonstrated high levels of TNF-a in different organs of rats between 6 to 72 hours after CLP-G18 procedure $(27,28,30,31,36,55,60)$. In this regard, we observed that in cardiopulmonary system, the levels of both aforementioned cytokines were more intensely increased when sepsis was induced using G-18 needles in comparison with the CLP-G21 group. This needs to be taken into consideration if future studies.

Mitochondrial membrane disturbance during ROS elevation results in formation activates caspase 9 and 3 with the natural consequence of cellular apoptosis (55). This programmed cell death process during CLP procedure is observed and reported in different tissues (mainly with gauge 18$)$ such as the kidney, lung, and brain $(35,39,40)$. Correspondingly, findings of our study revealed that the levels of the aforementioned caspases increased in the lung and heart with the higher intensity in CLP-G18 compared to CLP-G21.

A combination of ROS elevation and enhanced autophagy are responsible for elimination of abnormal proteins and promotion of organ failure-related apoptosis during sepsis. (61-65) Several studies have demonstrated that LC3-II/LC3I ratio increased in the liver, kidney, spleen and mesenteric nodes 6 to 72 hours after CLP procedure (with gauges 22 to 25) (66-69). Moreover, Hsaio et al showed an increase in LC3-Il levels in the renal tissues of rats after 3 hours after CLP-G18. Correspondingly, Escobar concluded that 8 hours after CLP-G22 procedure, LC3-II was increased in parallel with phosphorylated AMPK in kidney and liver (61). In this regard, we observed that the gene expression levels of mRNA of LC3-IIb and AMPK in both CLP-G18 and CLP-G21 was more pronouncedly increased in the heart and lung tissues of the animals in comparison with the sham group. Moreover, histopathological changes we observed confirmed the superiority of CLP-G18 in terms of provoking inflammation through demonstration of edematous and thickened lung tissues and myocardial cell necrosis. It is noteworthy however, that micrographs of CLP-G21 were similar to the sham group. These observations may support the hypothesis that in comparison with CLP-G21, CLP-G18 provokes oxidative stress reactions and cellular damage more pronouncedly in the cardiopulmonary system.

\section{Conclusion}

In conclusion, findings of this study demonstrated that CLP-G18 is superior to CLP-G21 in terms of the severity of sepsis induced in a rat model, and both the rise of inflammatory markers and the extent of the ensuing organ damage is greater in the group undergone the procedure with G18 needles. This study has identified cost-effective indicators to evaluate organ failure during sepsis. This should be considered by all investigators using G-18 in comparison with G21 to induce severe sepsis. It can be concluded that the choice of the needle size can have a great influence in the research outcomes, and this should be considered in design and implementation of CLP studies so that researchers can inflict the precise level of sepsis they intend to induce without waste of resources.

\section{Abbreviations}

CLP: Cecal Ligation and Puncture ICU: Intensive Care Unit ROS: Reactive Oxygen Substances AMPK: 5 ' Adenosine Monophosphate-Activated Protein Kinase LC3Ilb: Microtubule-associated protein 1A/1B-light chain 3b MPO: Myeloperoxidase HMGB1: High Mobility Group Box 1 TNF-a: Tumor Necrosis Factor alpha TUMS: Tehran University of Medical Science LPO: Lipid Peroxidation TBARS: Thiobarbituric Acid Reactive Substances MDA: Malondialdehyde FRAP: Ferric Reducing Antioxidant Power TTM: Total Thiol Molecules qRT-PCR: quantitative Real-Time Reverse

Page $11 / 20$ 
Transcription Polymerase chain reaction CBC: Complete Blood Count MPV: Mean Platelet Volume WBCs: White Blood Cells Hb: Hemoglobin PLR: Platelet to Lymphocyte Ratio

\section{Declarations}

\section{Acknowledgments}

We would like to thank the Tehran University of Medical Sciences for supporting this project.

\section{Authors' contributions}

T.D. performed all parts of the experiment and wrote the manuscript. S.M. participated in drafting and editing the article. M.B, M.G., H.H.A. helped to perform and design the experimental part of the study. A.N. and M.B., M.R., was involved in data analysis and interpretation. M.M. contributed to the concept, design, and financial support of the manuscript. All authors read and approved the final manuscript.

\section{Funding}

This study was supported by the Tehran University of Medical Sciences.

\section{Availability of data and material:}

The dataset used and analyzed during the current study is available from the corresponding author on reasonable request.

\section{Ethics approval and consent to participate:}

The protocol of the study was approved by the institutional ethical committee under code number IR.TUMS.VCR.REC.1396.2341.

\section{Consent for publication}

Not applicable.

\section{Competing interests:}

The authors declare that they have no competing interests.

\section{Author details}

a The Institute of Pharmaceutical Sciences (TIPS), Tehran University of Medical Sciences, Tehran, Iran. ${ }^{b}$ Department of Toxicology and Pharmacology, Tehran University of Medical Sciences, Tehran, Iran. ${ }^{\mathrm{C}}$ Pharmaceutical Sciences Research Center, Ardabil University of Medical Sciences, Ardabil, Iran. ${ }^{d}$ Department of Anesthesiology and Critical Care, Sina Hospital, Tehran University of Medical Sciences, Tehran, Iran. ${ }^{e}$ Department of Clinical Pharmacy, Tehran University of Medical Sciences, Tehran, Iran.

\section{References}

1. Drosatos K, Lymperopoulos A, Kennel PJ, Pollak N, Schulze PC, Goldberg IJ. Pathophysiology of sepsis-related cardiac dysfunction: driven by inflammation, energy mismanagement, or both? Current heart failure reports. 
2015;12(2):130-40.

2. Seymour CW, Gesten F, Prescott HC, Friedrich ME, Iwashyna TJ, Phillips GS, et al. Time to Treatment and Mortality during Mandated Emergency Care for Sepsis. The New England journal of medicine. 2017;376(23):2235-44.

3. Gonzalez MA, Ochoa CD. Multiorgan System Failure in Sepsis. Sepsis: Springer; 2018. p. 67-71.

4. Sun Y, Cai Y, Zang QS. Cardiac Autophagy in Sepsis. Cells. 2019;8(2).

5. Prescott HC, Angus DC. Enhancing Recovery From Sepsis: A Review. Jama. 2018;319(1):62-75.

6. Park I, Kim M, Choe K, Song E, Seo H, Hwang Y, et al. Neutrophils disturb pulmonary microcirculation in sepsisinduced acute lung injury. The European respiratory journal. 2019;53(3).

7. Sadowitz B, Roy S, Gatto LA, Habashi N, Nieman G. Lung injury induced by sepsis: lessons learned from large animal models and future directions for treatment. Expert review of anti-infective therapy. 2011;9(12):1169-78.

8. Rittirsch D, Huber-Lang MS, Flierl MA, Ward PA. Immunodesign of experimental sepsis by cecal ligation and puncture. Nature protocols. 2008;4(1):31.

9. Dejager L, Pinheiro I, Dejonckheere E, Libert C. Cecal ligation and puncture: the gold standard model for polymicrobial sepsis? Trends in microbiology. 2011;19(4):198-208.

10. Li L, Tan J, Miao Y, Lei P, Zhang Q. ROS and Autophagy: Interactions and Molecular Regulatory Mechanisms. Cellular and molecular neurobiology. 2015;35(5):615-21.

11. Aoki H, Kondo Y, Aldape K, Yamamoto A, Iwado E, Yokoyama T, et al. Monitoring autophagy in glioblastoma with antibody against isoform B of human microtubule-associated protein 1 light chain 3. Autophagy. 2008;4(4):46775 .

12. Faubert B, Boily G, Izreig S, Griss T, Samborska B, Dong Z, et al. AMPK is a negative regulator of the Warburg effect and suppresses tumor growth in vivo. Cell metabolism. 2013;17(1):113-24.

13. Ebong S, Call D, Nemzek J, Bolgos G, Newcomb D, Remick D. Immunopathologic alterations in murine models of sepsis of increasing severity. Infection and immunity. 1999;67(12):6603-10.

14. Wichterman KA, Baue AE, Chaudry IH. Sepsis and septic shock--a review of laboratory models and a proposal. J Surg Res. 1980;29(2):189-201.

15. Zapelini PH, Rezin GT, Cardoso MR, Ritter C, Klamt F, Moreira JC, et al. Antioxidant treatment reverses mitochondrial dysfunction in a sepsis animal model. Mitochondrion. 2008;8(3):211-8.

16. Shrum B, Anantha RV, Xu SX, Donnelly M, Haeryfar SM, McCormick JK, et al. A robust scoring system to evaluate sepsis severity in an animal model. BMC research notes. 2014;7(1):1-13.

17. Armstrong D BR. The analysis of free radicals, lipid peroxides, antioxidant enzymes and compounds related to oxidative stress as applied to the clinical chemistry laboratory. Adv Exp Med Biol. 1994;366:43-58.

18. Benzie IF SJ. The ferric reducing ability of plasma (FRAP) as a measure of "antioxidant power": the FRAP assay. Anal Biochem. 1996;239:70-6.

19. ML H. Measurement of protein thiol groups and glutathione in plasma. Methods in enzymology. 1994;233:380-5.

20. Mohammadi H KG, Rezayat SM, Dehpour AR, Shafiee H NS, et al. Benefit of nanocarrier of magnetic magnesium in rat malathion-induced toxicity and cardiac failure using non-invasive monitoring of electrocardiogram and blood pressure. Toxicol Ind Health. 2011;27:417-29.

21. Krawisz JE, Sharon P, Stenson WF. Quantitative assay for acute intestinal inflammation based on myeloperoxidase activity. Assessment of inflammation in rat and hamster models. Gastroenterology. 1984;87(6):1344-50. 
22. Rahimifard M, Navaei-Nigjeh M, Baeeri M, Maqbool F, Abdollahi M. Multiple protective mechanisms of alpha-lipoic acid in oxidation, apoptosis and inflammation against hydrogen peroxide induced toxicity in human lymphocytes. Molecular and cellular biochemistry. 2015;403(1-2):179-86.

23. Baeeri M, Momtaz S, Navaei-Nigjeh M, Niaz K, Rahimifard M, Ghasemi-Niri SF, et al. Molecular evidence on the protective effect of ellagic acid on phosalone-induced senescence in rat embryonic fibroblast cells. Food and chemical toxicology. 2017;100:8-23.

24. Husain-Syed F, McCullough PA, Birk HW, Renker M, Brocca A, Seeger W, et al. Cardio-Pulmonary-Renal Interactions: A Multidisciplinary Approach. Journal of the American College of Cardiology. 2015;65(22):2433-48.

25. Goto M, Samonte V, Ravindranath T, Sayeed MM, Gamelli RL. Burn injury exacerbates hemodynamic and metabolic responses in rats with polymicrobial sepsis. Journal of burn care \& research : official publication of the American Burn Association. 2006;27(1):50-9.

26. Liu H, Wu J, Yao JY, Wang H, Li ST. The Role of Oxidative Stress in Decreased Acetylcholinesterase Activity at the Neuromuscular Junction of the Diaphragm during Sepsis. Oxid Med Cell Longev. 2017;2017:1-6.

27. Sener G, Sehirli O, Cetinel S, Ercan F, Yuksel M, Gedik N, et al. Amelioration of sepsis-induced hepatic and ileal injury in rats by the leukotriene receptor blocker montelukast. Prostaglandins, leukotrienes, and essential fatty acids. 2005;73(6):453-62.

28. Sener G, Toklu H, Ercan F, Erkanli G. Protective effect of beta-glucan against oxidative organ injury in a rat model of sepsis. Int Immunopharmacol. 2005;5(9):1387-96.

29. Sener G, Toklu H, Kapucu C, Ercan F, Erkanli G, Kacmaz A, et al. Melatonin protects against oxidative organ injury in a rat model of sepsis. Surg Today. 2005;35(1):52-9.

30. Xiao X, Yang M, Sun D, Sun S. Curcumin protects against sepsis-induced acute lung injury in rats. J Surg Res. 2012;176(1):e31-9.

31. Chen HH, Lin KC, Wallace CG, Chen YT, Yang CC, Leu S, et al. Additional benefit of combined therapy with melatonin and apoptotic adipose-derived mesenchymal stem cell against sepsis-induced kidney injury. Journal of pineal research. 2014;57(1):16-32.

32. Zhu W, Lu Q, Wan L, Feng J, Chen HW. Sodium tanshinone II A sulfonate ameliorates microcirculatory disturbance of small intestine by attenuating the production of reactie oxygen species in rats with sepsis. Chinese journal of integrative medicine. 2016;22(10):745-51.

33. Gonzalez AS, Elguero ME, Finocchietto P, Holod S, Romorini L, Miriuka SG, et al. Abnormal mitochondrial fusionfission balance contributes to the progression of experimental sepsis. Free radical research. 2014;48(7):769-83.

34. Wang P, Huang J, Li Y, Chang R, Wu H, Lin J, et al. Exogenous Carbon Monoxide Decreases Sepsis-Induced Acute Kidney Injury and Inhibits NLRP3 Inflammasome Activation in Rats. International journal of molecular sciences. 2015;16(9):20595-608.

35. Cinel I, Ark M, Dellinger P, Karabacak T, Tamer L, Cinel L, et al. Involvement of Rho kinase (ROCK) in sepsis-induced acute lung injury. Journal of thoracic disease. 2012;4(1):30-9.

36. Fujimura N, Sumita S, Narimatsu E, Shichinohe Y, Iwasaki H, Watababe H, et al. Diaphragmatic contractility in septic peritonitis-Role of free radical scavengers. Critical Care Medicine. 1999;27(1):41A.

37. Gerin F, Sener U, Erman H, Yilmaz A, Aydin B, Armutcu F, et al. The Effects of Quercetin on Acute Lung Injury and Biomarkers of Inflammation and Oxidative Stress in the Rat Model of Sepsis. Inflammation. 2016;39(2):700-5.

38. Hsu D-Z, Chien S-P, Li Y-H, Chuang Y-C, Chang Y-C, Liu M-Y. Sesame oil attenuates hepatic lipid peroxidation by inhibiting nitric oxide and superoxide anion generation in septic rats. Journal of Parenteral and Enteral Nutrition. 2008;32(2):154-9.

Page $14 / 20$ 
39. Olguner CG, Koca U, Altekin E, Ergur BU, Duru S, Girgin P, et al. Ischemic preconditioning attenuates lipid peroxidation and apoptosis in the cecal ligation and puncture model of sepsis. Experimental and therapeutic medicine. 2013;5(6):1581-8.

40. Zhou J, Chen Y, Huang GQ, Li J, Wu GM, Liu L, et al. Hydrogen-rich saline reverses oxidative stress, cognitive impairment, and mortality in rats submitted to sepsis by cecal ligation and puncture. J Surg Res. 2012;178(1):390400.

41. Hu JF, Wang HX, Li HH, Hu J, Yu Y, Gao Q. Inhibition of ALDH2 expression aggravates renal injury in a rat sepsis syndrome model. Experimental and therapeutic medicine. 2017;14(3):2249-54.

42. Eberhardt A, Lessig F, Schreiter K, Kellner N, Fuchs M, Sablotzki A, et al. Mean platelet volume (MPV) is an outcome marker in sepsis patients. International Journal of Infectious Diseases. 2012;16:e218.

43. Kim CH, Kim SJ, Lee MJ, Kwon YE, Kim YL, Park KS, et al. An increase in mean platelet volume from baseline is associated with mortality in patients with severe sepsis or septic shock. PLoS One. 2015;10(3):1-13.

44. Kitazawa T, Yoshino $\mathrm{Y}$, Tatsuno $\mathrm{K}$, Ota $\mathrm{Y}$, Yotsuyanagi $\mathrm{H}$. Changes in the mean platelet volume levels after bloodstream infection have prognostic value. Internal Medicine. 2013;52(13):1487-93.

45. Van der Lelie J, Von dem Borne AK. Increased mean platelet volume in septicaemia. Journal of clinical pathology. 1983;36(6):693-6.

46. Yilmaz Z, Eralp O, Ilcol YO. Evaluation of platelet count and its association with plateletcrit, mean platelet volume, and platelet size distribution width in a canine model of endotoxemia. Veterinary clinical pathology. 2008;37(2):159-63.

47. Drechsler S, Weixelbaumer KM, Weidinger A, Raeven P, Khadem A, Redl H, et al. Why do they die? Comparison of selected aspects of organ injury and dysfunction in mice surviving and dying in acute abdominal sepsis. Intensive care medicine experimental. 2015;3(1):1-21.

48. Hyde SR, McCallum RE. Lipopolysaccharide-tumor necrosis factor-glucocorticoid interactions during cecal ligation and puncture-induced sepsis in mature versus senescent mice. Infect Immun. 1992;60(3):976-82.

49. Liaw WJ, Chen TH, Lai ZZ, Chen SJ, Chen A, Tzao C, et al. Effects of a membrane-permeable radical scavenger, Tempol, on intraperitoneal sepsis-induced organ injury in rats. Shock. 2005;23(1):88-96.

50. Maitra SR, Wojnar MM, Lang CH. Alterations in tissue glucose uptake during the hyperglycemic and hypoglycemic phases of sepsis. Shock. 2000;13(5):379-85.

51. Kutlucan L, Kutlucan A, Basaran B, Dagli M, Basturk A, Kozanhan B, et al. The predictive effect of initial complete blood count of intensive care unit patients on mortality, length of hospitalization, and nosocomial infections. Eur Rev Med Pharmacol Sci. 2016;20(8):1467-73.

52. Turkmen K, Erdur FM, Ozcicek F, Ozcicek A, Akbas EM, Ozbicer A, et al. Platelet-to-lymphocyte ratio better predicts inflammation than neutrophil-to-lymphocyte ratio in end-stage renal disease patients. Hemodialysis International. 2013;17(3):391-6.

53. Tsao CM, Jhang JG, Chen SJ, Ka SM, Wu TC, Liaw WJ, et al. Adjuvant potential of selegiline in attenuating organ dysfunction in septic rats with peritonitis. PLoS One. 2014;9(9):1-9.

54. Yang S, Zhou M, Chaudry IH, Wang P. Novel approach to prevent the transition from the hyperdynamic phase to the hypodynamic phase of sepsis: role of adrenomedullin and adrenomedullin binding protein-1. Ann Surg. 2002;236(5):625-33.

55. Zhai X, Yang Z, Zheng G, Yu T, Wang P, Liu X, et al. Lactate as a Potential Biomarker of Sepsis in a Rat Cecal Ligation and Puncture Model. Mediators Inflamm. 2018;2018:1-9. 
56. Abdulmahdi W, Patel D, Rabadi MM, Azar T, Jules E, Lipphardt M, et al. HMGB1 redox during sepsis. Redox Biol. 2017; 13:600-7.

57. Léger T, Charrier A, Moreau C, Hininger-Favier I, Mourmoura E, Rigaudière JP, et al. Early sepsis does not stimulate reactive oxygen species production and does not reduce cardiac function despite an increased inflammation status. Physiological Reports. 2017;5(13):1-12.

58. Stevens NE, Chapman MJ, Fraser CK, Kuchel TR, Hayball JD, Diener KR. Therapeutic targeting of HMGB1 during experimental sepsis modulates the inflammatory cytokine profile to one associated with improved clinical outcomes. Scientific reports. 2017;7(1):1-14.

59. Lin WJ, Yeh WC. Implication of Toll-like receptor and tumor necrosis factor alpha signaling in septic shock. Shock. 2005;24(3):206-9.

60. Chang CL, Leu S, Sung HC, Zhen YY, Cho CL, Chen A, et al. Impact of apoptotic adipose-derived mesenchymal stem cells on attenuating organ damage and reducing mortality in rat sepsis syndrome induced by cecal puncture and ligation. Journal of translational medicine. 2012;10:1-14.

61. Escobar DA, Botero-Quintero AM, Kautza BC, Luciano J, Loughran P, Darwiche S, et al. Adenosine monophosphateactivated protein kinase activation protects against sepsis-induced organ injury and inflammation. J Surg Res. 2015;194(1):262-72.

62. Nishida K, Kyoi S, Yamaguchi O, Sadoshima J, Otsu K. The role of autophagy in the heart. Cell Death Differ. 2009;16(1):31-8.

63. Zaha VG, Young LH. AMP-activated protein kinase regulation and biological actions in the heart. Circulation research. 2012;111(6):800-14.

64. Mizumura K, Cloonan S, Choi ME, Hashimoto S, Nakahira K, Ryter SW, et al. Autophagy: friend or foe in lung disease? Annals of the American Thoracic Society. 2016;13(Supplement 1):S40-S7.

65. Mungai PT, Waypa GB, Jairaman A, Prakriya M, Dokic D, Ball MK, et al. Hypoxia triggers AMPK activation through reactive oxygen species-mediated activation of calcium release-activated calcium channels. Mol Cell Biol. 2011;31(17):3531-45.

66. Hsieh CH, Pai PY, Hsueh HW, Yuan SS, Hsieh YC. Complete induction of autophagy is essential for cardioprotection in sepsis. Ann Surg. 2011;253(6):1190-200.

67. Lee S, Lee SJ, Coronata AA, Fredenburgh LE, Chung SW, Perrella MA, et al. Carbon monoxide confers protection in sepsis by enhancing beclin 1-dependent autophagy and phagocytosis. Antioxidants \& redox signaling. 2014;20(3):432-42.

68. Watanabe E, Muenzer JT, Hawkins WG, Davis CG, Dixon DJ, McDunn JE, et al. Sepsis induces extensive autophagic vacuolization in hepatocytes: a clinical and laboratory-based study. Laboratory investigation; a journal of technical methods and pathology. 2009;89(5):549-61.

69. Takahashi W, Hatano H, Hirasawa H, Oda S. Protective role of autophagy in mouse cecal ligation and punctureinduced sepsis model. Critical Care. 2013;17(2):1-13.

\section{Figures}




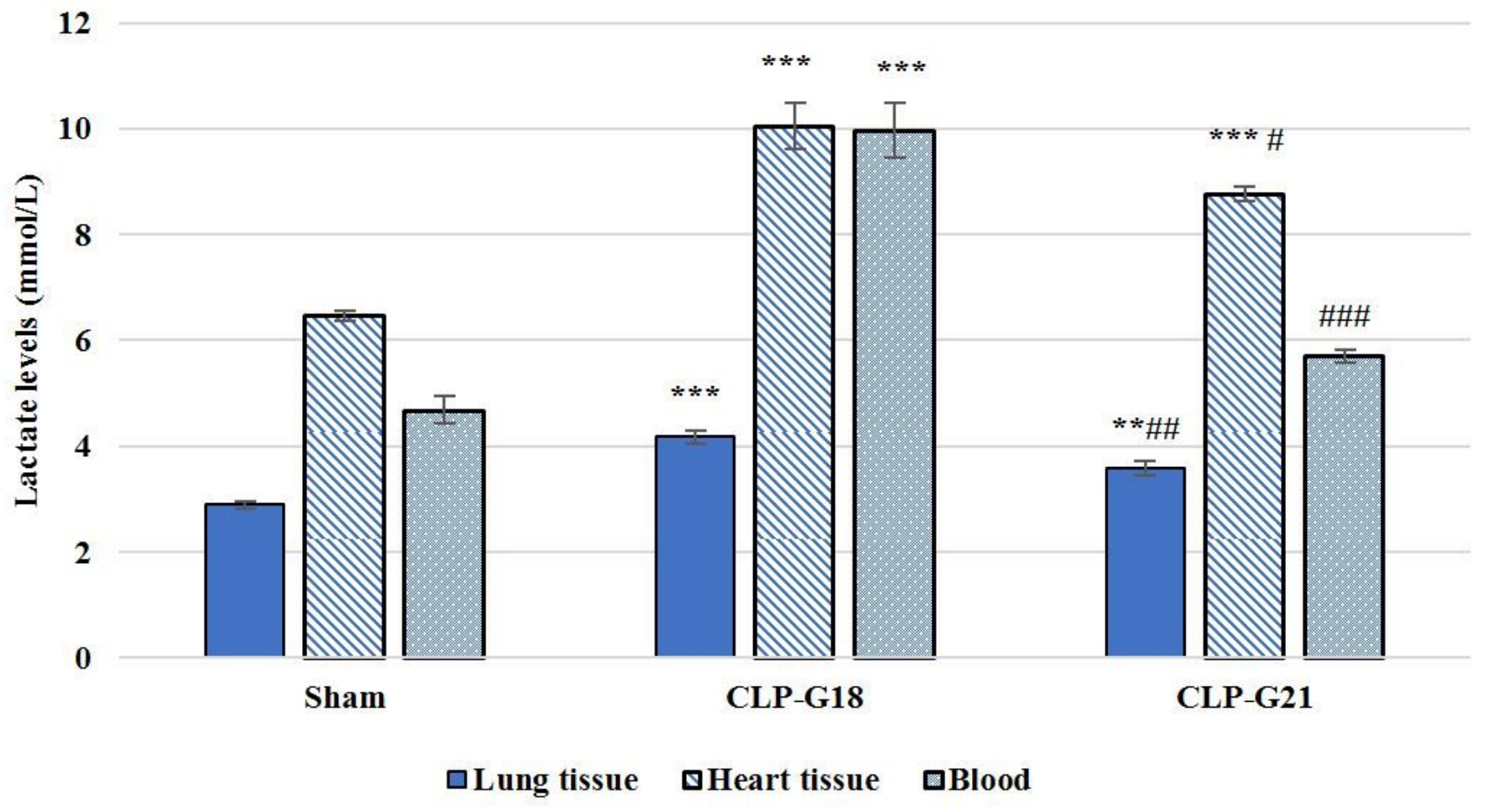

Figure 1

Effect of gauge 18 and gauge 21 on Cecal ligation and puncture (CLP) on lactate levels in heart, lung tissues and blood. Results are expressed as mean \pm SEM for six animals in each group. ${ }^{\star \star *}$ : significant difference from sham group at $P<0.001$, **: significant difference from sham group at $P<0.01$, \#\#\#: significant difference from CLPgauge18 group at $P<0.001$, \#\#: significant difference from CLP-gauge18 group at $P<0.01$, \#: significant difference from CLP-gauge18 group at $\mathrm{P}<0.05$.
A: TNF-a
B: HMGB1
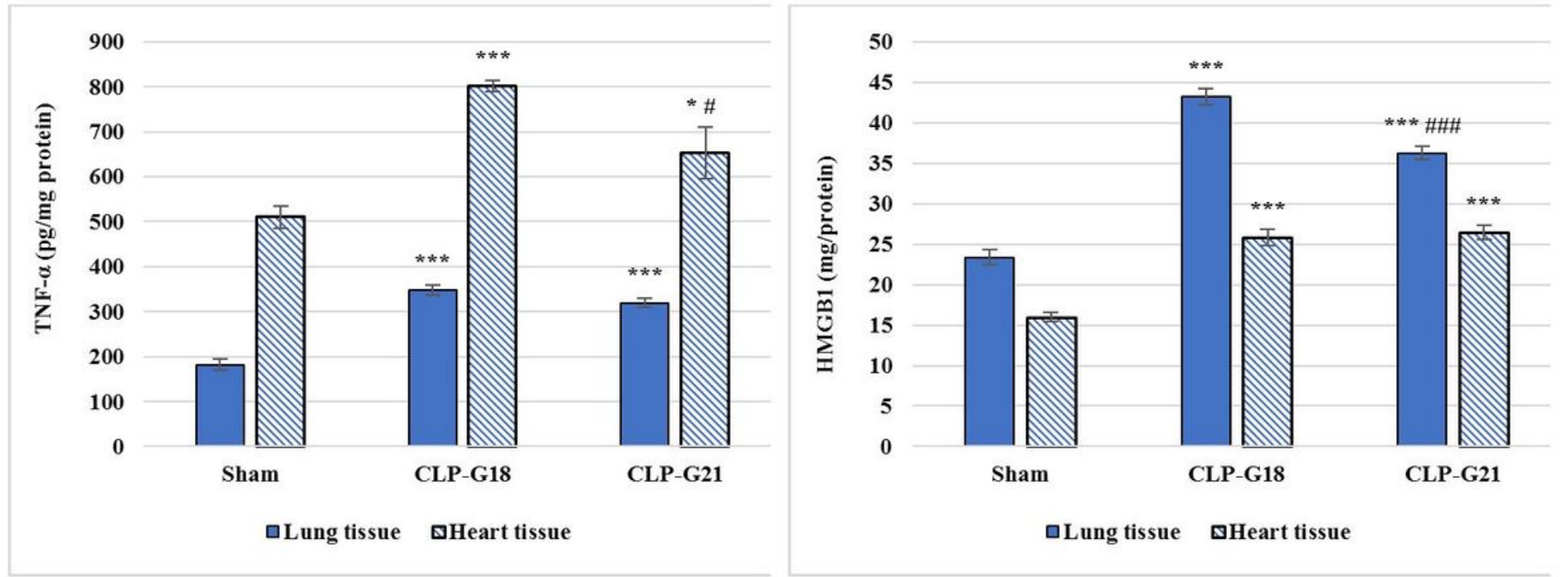

Figure 2 
Effect of gauge 18 and gauge 21 on Cecal ligation and puncture (CLP) on proinflammatory cytokines. A: Tumor Necrosis Factor-a (TNF-a), B: High Mobility Group Box 1 (HMGB1) in myocardial and pulmonary tissues. Results are expressed as mean \pm SEM for six animals in each group. ***: significant difference from sham group at $P<0.001$, *: significant difference from sham group at $P<0.05$, \#\#\#: significant difference from CLP-gauge18 group at $P<0.001$, \#: significant difference from CLP-gauge18 group at $\mathrm{P}<0.05$.

\section{A: AMPK}

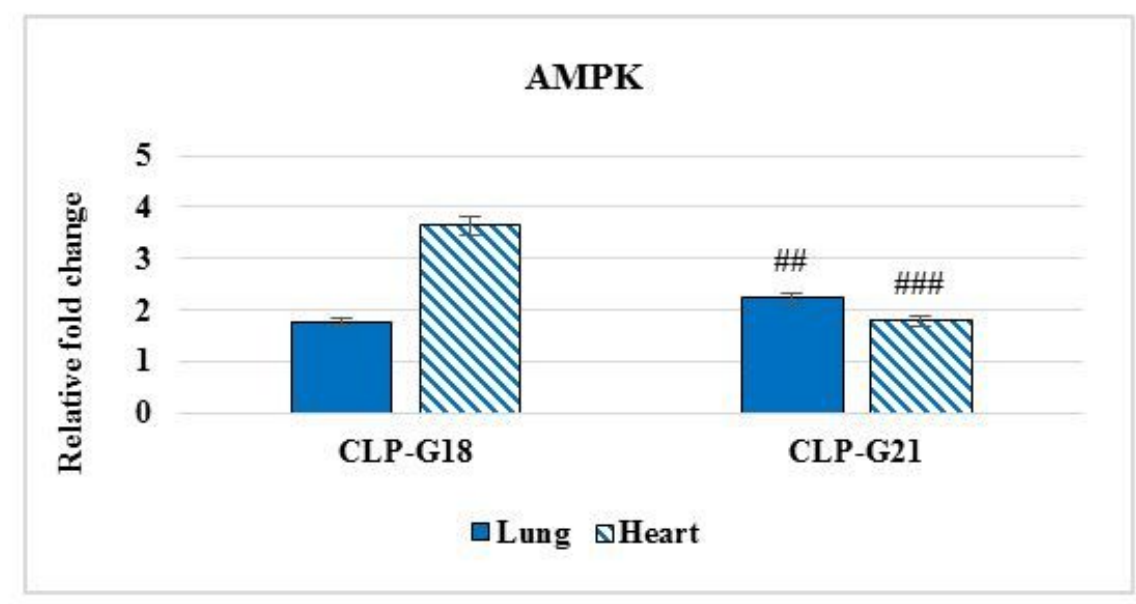

\section{B: LC3חb}

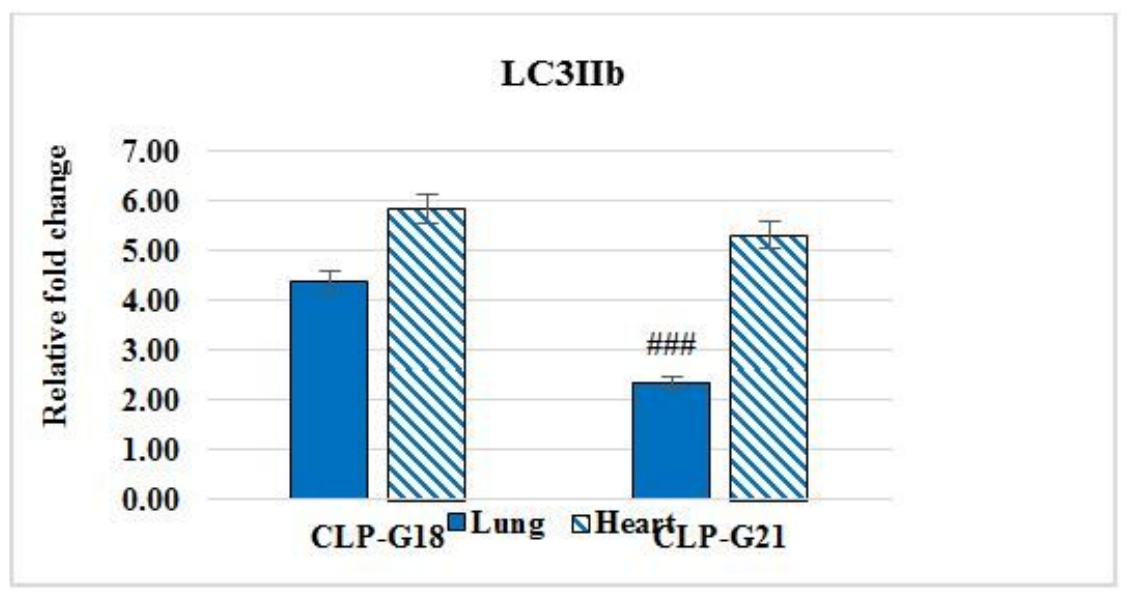

Figure 3

Effect of gauge 18 and gauge 21 on Cecal ligation and puncture (CLP) on mRNA expression pattern of LC3Ilb (Microtubule-associated protein 1 light chain 3) and AMPK (AMP-activated protein kinase) genes in myocardial and pulmonary tissues. Results are expressed as mean \pm SEM for six animals in each group. \#\#\#: significant difference from CLP-gauge18 group at $P<0.001$, \#\#: significant difference from CLP-gauge18 group at $P<0.01$. 


\section{A: MPV}

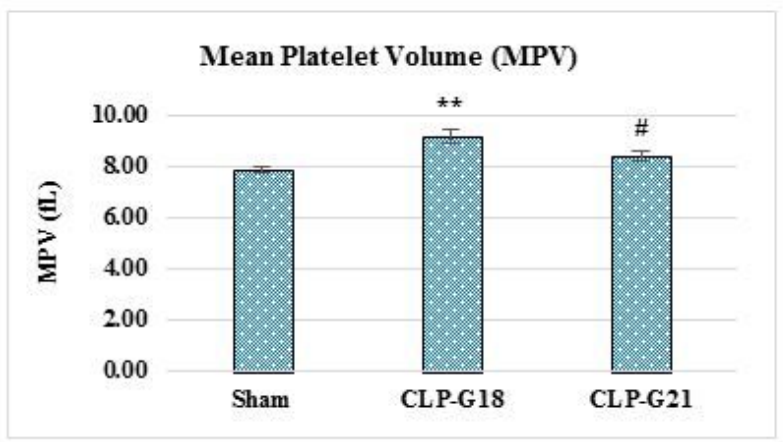

\section{B: PLR}

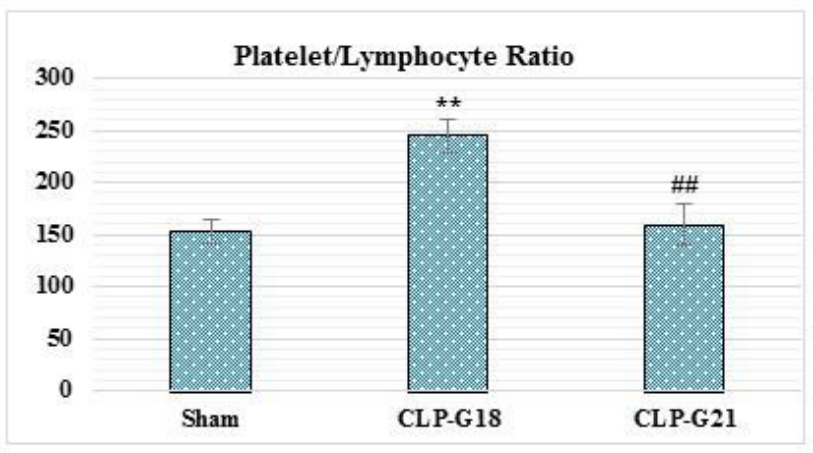

\section{C: Blood glucose levels}

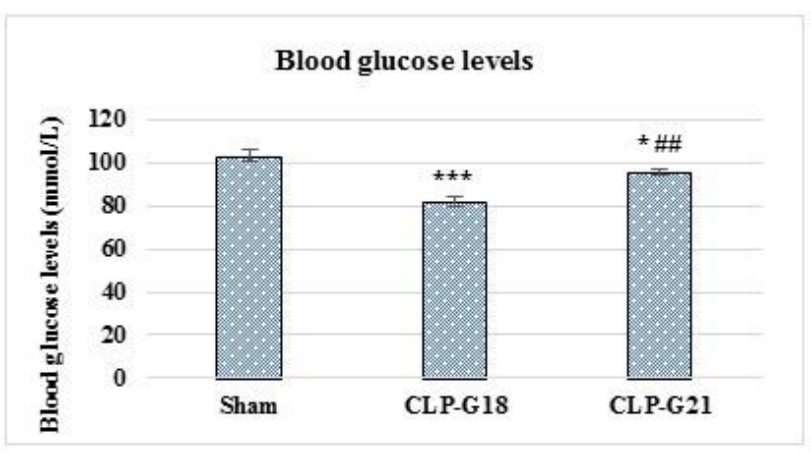

\section{Figure 4}

Effect of gauge 18 and gauge 21 on Cecal ligation and puncture (CLP) on blood markers. A: MPV (mean platelet volume), B: PLR (platelet to lymphocyte ratio), C: Blood glucose levels. Results are expressed as mean \pm SEM for six animals in each group. $* \star *$ : significant difference from sham group at $P<0.001$, $* *$ : significant difference from sham group at $P<0.01$, *: significant difference from sham group at $P<0.05$, \#\#: significant difference from CLP-gauge18 group at $P<0.01$, \#: significant difference from CLP-gauge18 group at $P<0.05$. 


\section{Lung-X200 Heart-X200}
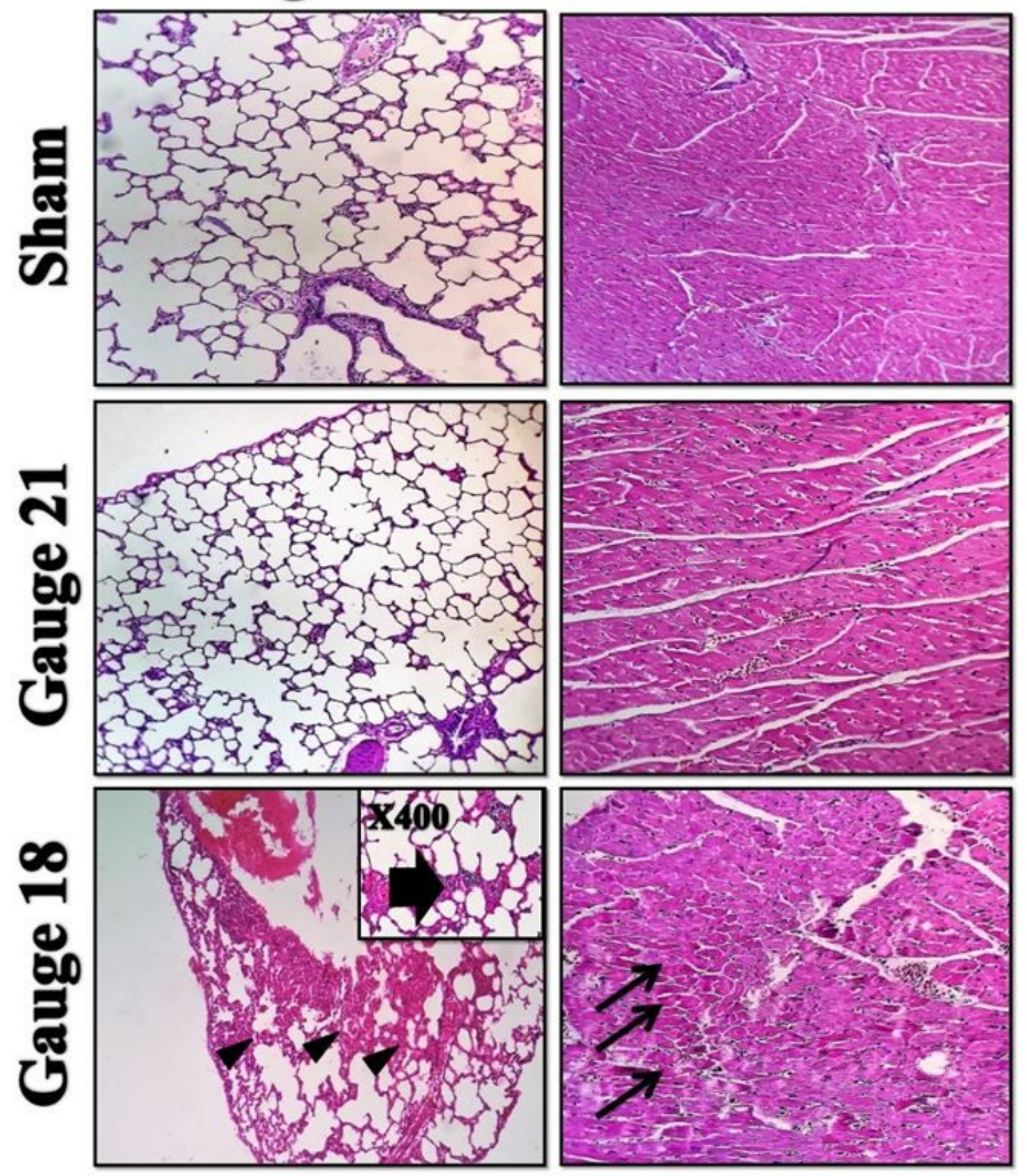

Figure 5

The histopathology of the lung and heart in different groups. Arrow heads: hyperplasia of pneumocyte type A, Thick arrow: perivascular inflammation and infiltration of inflammatory cells, Thin arrows: myocardial necrosis. 